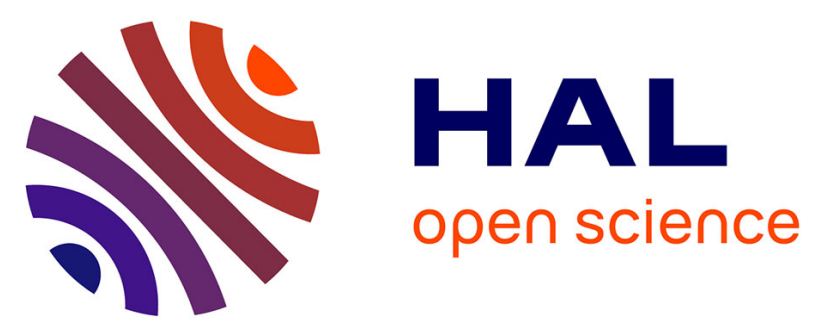

\title{
Application of Multipreconditioned Iterative Algorithms in Dual Domain Decomposition Methods for Structural Dynamics
}

\author{
Michael C. Leistner, Pierre Gosselet, Daniel J. Rixen
}

\section{To cite this version:}

Michael C. Leistner, Pierre Gosselet, Daniel J. Rixen. Application of Multipreconditioned Iterative Algorithms in Dual Domain Decomposition Methods for Structural Dynamics. Proceedings in applied mathematics and mechanics (88th GAMM annual meeting), 2017, Weimar, Germany. 10.1002/pamm.201710134 . hal-01615876

\author{
HAL Id: hal-01615876 \\ https://hal.science/hal-01615876
}

Submitted on 13 Jun 2018

HAL is a multi-disciplinary open access archive for the deposit and dissemination of scientific research documents, whether they are published or not. The documents may come from teaching and research institutions in France or abroad, or from public or private research centers.
L'archive ouverte pluridisciplinaire HAL, est destinée au dépôt et à la diffusion de documents scientifiques de niveau recherche, publiés ou non, émanant des établissements d'enseignement et de recherche français ou étrangers, des laboratoires publics ou privés.

\section{(ㅇ)(1) $\$$}

Distributed under a Creative Commons Attribution - NonCommercial - NoDerivatives| 4.0 


\title{
Application of Multipreconditioned Iterative Algorithms in Dual Domain Decomposition Methods for Structural Dynamics
}

\author{
Michael C. Leistner ${ }^{1,4}$, Pierre Gosselet ${ }^{2}$, Daniel J. Rixen ${ }^{1}$ \\ ${ }^{1}$ Technical University of Munich, Chair of Applied Mechanics, Boltzmannstr. 15, \\ D-85748 Garching, Germany \\ ${ }^{2}$ LMT-Cachan/ENS-Cachan, CNRS, Université Paris-Saclay, 61 avenue du président Wilson, \\ Cachan 94235, France
}

\begin{abstract}
We apply a multipreconditioned domain decomposition method based on Finite Element Tearing and Interconnecting to linear structural dynamics with highly heterogeneous material properties. A recently published method to build and select the multiple directions is presented and applied. We hint at possible problems when localized phenomena are considered and present a new simple measure that extends the selection process in existing algorithms. We show numerical results and conclude how to effectively prevent a possible degeneration of the minimization space basis.
\end{abstract}

KEY WORDS: Feti; adaptive; multipreconditioned; dynamics; minimization space basis; degeneration

\section{Multipreconditioned FETI for Dynamic Structural Problems}

We solve a linear problem of structural dynamics, discretized using FEM. The dual domain decomposition method FETI is applied, decomposing the domain in $N_{s}$ substructures. It was originally published in [1] and applied to dynamic problems in [2]. Application of the Newmark Beta timestepping scheme yields the final equations $\boldsymbol{D}^{s} \ddot{\boldsymbol{u}}^{s, n+1}=\boldsymbol{g}^{s, n+1}-\boldsymbol{B}^{s T} \boldsymbol{\lambda}$ to be solved for every substructure to compute the acceleration at time $t_{n+1}$, together with the interface compatibility $r=$ $\sum_{s=1}^{N_{s}} \boldsymbol{B}^{s} \ddot{\boldsymbol{u}}^{s}=\mathbf{0}$ imposed on the accelerations. These linear substructure systems that are coupled by their interface forces, here denoted by Lagrange Multipliers $\lambda$, can be solved with any FETI method. All those methods have in common that they minimize the interface error $\boldsymbol{r}=\boldsymbol{d}-\boldsymbol{F} \boldsymbol{\lambda}$ iteratively by applying a conjugate gradient algorithm to the system

$$
\boldsymbol{F} \boldsymbol{\lambda}=\boldsymbol{d} \quad \boldsymbol{F}=\sum_{s} \boldsymbol{F}^{s}=\sum_{s} \boldsymbol{B}^{s} \boldsymbol{D}^{s-1} \boldsymbol{B}^{s T} \quad \boldsymbol{H}=\sum_{s} \boldsymbol{H}^{s}=\sum_{s} \widetilde{\boldsymbol{B}}^{s} \boldsymbol{D}_{S, b}^{s} \widetilde{\boldsymbol{B}}^{s T}
$$

using the so called Dirichlet preconditioner $\boldsymbol{H}$ which uses the Schur complements of the substructure operators on the interface $\boldsymbol{D}_{S, b}^{s}$ to estimate the adaption of interface forces. While $\boldsymbol{B}^{s}$ are signed boolean assembly matrices, $\widetilde{\boldsymbol{B}}^{s}$ denote scaled variants of those, accounting for heterogeneities. The typical preconditioners such as (1) all consist of a sum of local contributions which could also be taken as separate search directions to find the optimal solution within the subspace they span. While the idea was already proposed in [3], it was thoroughly developed and published in [4]. It was generalized further in [5] to a method that selects adaptively which contributions should be considered as independent search directions. Based on local estimates of the convergence rate, the following criterion is presented in [5]. When in CG step $i$ the columns of $\boldsymbol{P}_{i}$ have been used as independent directions with the step lengths $\boldsymbol{\alpha}_{i}$, the contributions of all substructures matching the $\tau$-criterion $\Theta_{i}^{s}=\left(\boldsymbol{\alpha}_{i}^{T} \boldsymbol{P}_{i}^{T} \boldsymbol{F}^{s} \boldsymbol{P}_{i} \boldsymbol{\alpha}_{i}\right) /\left(\boldsymbol{r}_{i+1}^{T} \boldsymbol{H}^{s} \boldsymbol{r}_{i+1}\right)<\tau$ are selected as separate directions for the next CG step while all others are summed up to form one single direction. The method has been applied to static problems and shows an improvement in efficiency for ill-conditioned systems compared to classical FETI methods. We apply the procedure to a dynamic problem shown in Fig. 2 with highly heterogeneous material properties which are typically hard to solve for this class of algorithms. The nodes on the left side are fixed and a shock load is applied in the first time step in the middle of the upper side. The adaptive selection algorithm is driven by $\tau=0.1$ in all calculations.

\section{Procedures to Prevent Degeneration of Basis}

The very localized effects in this example lead to local search direction contributions that differ in size by several orders of magnitude. To be able to consider these effects properly, we express the $\tau$-criterion in terms of a localized search direction energy $E_{p}$, and a localized error energy $E_{r}$. Further we introduce a new measure and an additional selection criterion based on the relative localized error energy $e_{r}$.

$$
\Theta_{i}^{s}=\frac{E_{p, i}^{s}}{E_{r, i+1}^{s}}=\frac{\boldsymbol{\alpha}_{i}^{T} \boldsymbol{P}_{i}^{T} \boldsymbol{F}^{s} \boldsymbol{P}_{i} \boldsymbol{\alpha}_{i}}{\boldsymbol{r}_{i+1}^{T} \boldsymbol{H}^{s} \boldsymbol{r}_{i+1}} \quad e_{r, i+1}^{s}=\frac{E_{r, i+1}^{s}}{\sum_{s} E_{r, i+1}^{s}}>\epsilon_{r}
$$

Because of limited machine precision, it becomes numerically impossible to simultaneously minimize the error in two directions with extremely different magnitudes. However, although the local error on a substructure is already within an acceptable

\footnotetext{
${ }^{4}$ Correspondence to: Michael C. Leistner, m.leistner@tum.de
} 
range, the $\tau$-criterion may select its contribution as separate direction because $E_{p}$ is smaller than $E_{r}$ by several orders of magnitude. As suggested in [4], we use a rank revealing factorization for solving the minimization problem with a tolerance of $\epsilon_{L D L}$. Additionally, we apply the criterion (2) to detect directions that do not significantly contribute to the residual. While the directions considered linearly dependent by the rank revealing factorization are removed completely from the minimization space, the directions not fulfilling the $\epsilon_{r}$ criterion are summed up as if they had not been selected by the $\tau$-criterion, i.e. they are "deselected".

\begin{tabular}{|c|c|c|c|}
\hline \multicolumn{4}{|c|}{$\begin{array}{l}\text { 10th Ef. } f_{10}=59.3 \mathrm{~Hz} \\
\text { time step } h=4 \times 10^{-4} \mathrm{~s}\end{array}$} \\
\hline & $\tau$ & $\epsilon_{L D L}$ & $\epsilon_{r}$ \\
\hline R6 & 0.1 & $10^{-6}$ & - \\
\hline R11 & 0.1 & $10^{-11}$ & - \\
\hline $\mathrm{R} \epsilon_{m}$ & 0.1 & $\epsilon_{m}$ & - \\
\hline R11-e6 & 0.1 & $10^{-11}$ & $10^{-6}$ \\
\hline $\mathrm{R} \epsilon_{m}$-e6 & 0.1 & $\epsilon_{m}$ & $10^{-6}$ \\
\hline
\end{tabular}
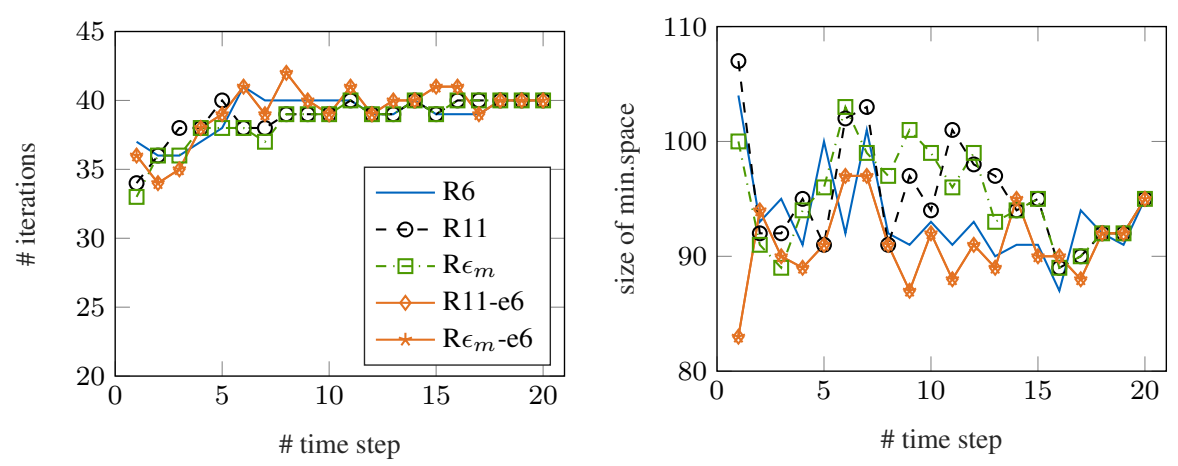

Figure 1. The left graph shows that all compared variants solve the problem. $\epsilon_{m}$ denotes the machine precision which in this case is $2.3 \times 10^{-16}$. The right graph shows the accumulated size of the minimization space within one time step.
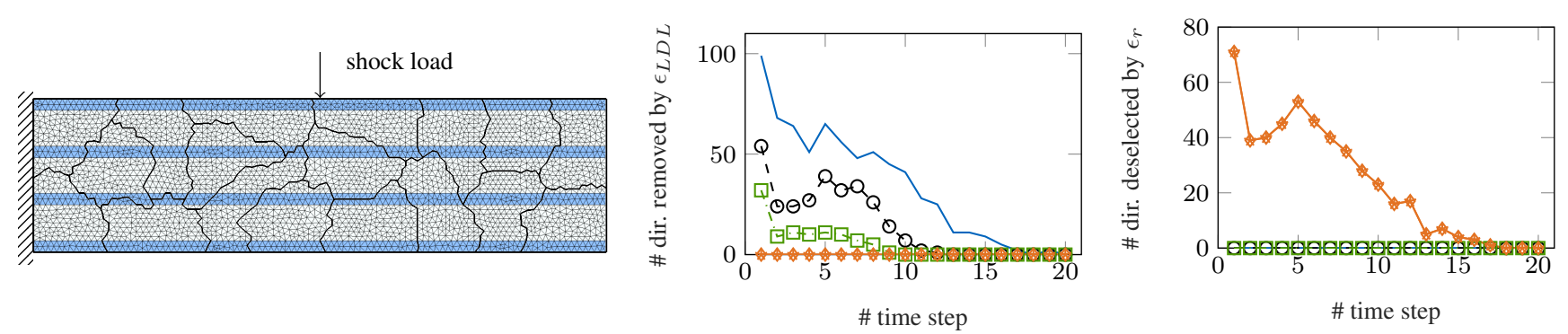

Figure 2. The figure on the left shows the applied shock load and the material distribution in the structure as well as its decomposition into 18 substructures by the METIS algorithm. The stripes exhibit a stiffness ratio of $10^{4}$ and a density ratio of $10^{1}$. The left graph shows the number of directions removed by the rank revealing factorization, the right one the number of directions pushed back into the summed direction by the $\epsilon_{r}$-criterion.

First of all, the numerical results show that this effect may be limited to the first few time steps of a dynamic problem, but is always likely to occur when a static equilibrium position is chosen as initial state. The intuitive selection for $\epsilon_{L D L}$ as machine precision $\epsilon_{m}$ solves the problem but in general results in the largest minimization space. Further more, larger values for $\epsilon_{L D L}$ or $\epsilon_{r}$, leading to a high number of directions being removed completely or only taken into account as their sum, result in a similar performance as smaller values. We explain this by the fact that both critera work with relative tolerances, thus by applying more strict criteria, the directions dropped in early iterations are simply taken into account in later, additional iterations. Finally, the rank revealing factorization and the $\epsilon_{r}$-criterion lead to very similar results while the use of a strict $\epsilon_{r}$ criterion already prevents a degeneration of the minimization space basis effectively and without destabilizing the algorithm. Future work should focus on the optimal choice of the tolerances and on quantifying possible efficiency improvements.

\section{References}

[1] C. Farhat and F.-X. Roux, A Method of Finite Element Tearing and Interconnecting and its Parallel Solution Algorithm. Int. J. Numer. Meth. Engng 32, 1205-1227 (1991)

[2] C. Farhat, L. Crivelli and F.-X. Roux, A transient FETI methodology for large-scale parallel implicit computations in structural mechanics. Int. J. Numer. Meth. Engng 37, 1945-1975 (1994)

[3] D. J. Rixen, Substructuring and Dual Methods in Structural Analysis. PhD thesis. Publications de la Faculté des Sciences Appliquées, 175, Université de Liège, Belgium, 1997

[4] P. Gosselet, D. J. Rixen, F.-X. Roux and N. Spillane, Simultaneous FETI and block FETI: Robust domain decomposition with multiple search directions. Int. J. Numer. Meth. Engng 104, 905-927 (2015)

[5] N. Spillane, An Adaptive MultiPreconditioned Conjugate Gradient Algorithm. SIAM J. Sci. Comput. 38, A1896-A1918 (2016) 